\title{
Preferred Frequencies for Coupling of Seismic Waves and Vibrating Tall Buildings
}

\author{
Darko Volkov and Sergey Zheltukhin *
}

November 11, 2018

\begin{abstract}
We study a model for the so called "city effect" in which an earthquake can be locally enhanced by the collective response of tall buildings in a large city. We use a set of equations coupling vibrations in buildings to motion under the ground. These equations were previously studied exclusively in the case of a finite set of identical, equally spaced, buildings. These two restrictions are lifted in this paper. We may now simulate geometries involving infinitely many buildings as long as an initial pattern of buildings is repeated. Our new method using periodic domains and periodic Green's functions yields much faster computations. This is the main reason why we are now able to study systems of buildings of variable height, mass, and rigidity. We show how solving for the wavenumber in a non-linear equation involving the integral of a function solution to an adequate integral equation, we are able to find resonant frequencies coupling seismic waves and vibrating tall buildings. Interestingly, in the case of non identical buildings, our simulations indicate that the response to this coupling phenomenon may differ drastically from one building to another.
\end{abstract}

Keywords: Seismic waves, city effect, integral equations, periodic domains.

\section{Introduction}

The traditional approach to evaluating seismic risk in urban areas is to consider seismic waves in the underground as the only cause for motion above ground. In earlier studies, seismic wave propagation was evaluated in an initial step and in a second step impacts on man made structures were inferred. However, observational evidence has since then suggested that when an earthquake strikes a large city, seismic activity may in turn be altered by the response of the buildings. This phenomenon is referred to as the "city-effect" and has been studied by many authors, see [3, 8, 5 .

Many occurrences of this city-effect have been documented. In 1970, vibrations of the Millikan library on the Caltech campus triggered by roof actuators were registered by seismographs located a few kilometers away (see [3] for a related phenomenon recorded in 2002). On multiple instances a shock wave formed as the NASA space shuttle was returning in the atmosphere and as a result many seismic stations near Edwards Air Force Base, California

\footnotetext{
*Department of Mathematical Sciences, Worcester Polytechnic Institute, Worcester MA 01609 (darko@wpi.edu, sergey@wpi.edu)
} 
( see [8]), recorded tremors. In particular, a shock wave created by the re-entry of the Columbia space shuttle in the atmosphere hit high buildings in Los Angeles and induced seismic waves which were recorded by seismographs in Pasadena, situated about 15 kilometers away. During the 2001 attacks on the Twin Towers in New York City, shortly after the towers were hit, tremors were recorded tens of kilometers away. It is hypothesized that in these three examples the transmission of waves to the underground occurred because the natural vibration frequencies of the buildings occurred to be very close to the frequencies of the ground layers in these areas. Possibly, the most significant observation of the cityeffect happened during the Michoacan earthquake that struck Mexico city in 1985 (see, for example, [7). Classical models and computational methods failed to explain all the seismic features recorded during that event. This led Wirgin and Bard in [12] to suggest that ground motion may be significantly altered by the presence of buildings and this effect may have been enhanced in Mexico City since it is such a densely urbanized area. Bard et al. showed experimentally in 2] that this hypothesis certainly has some relevance and is worth exploring further.

More recently, 6], Ghergu and Ionescu have derived a model for the city effect based on the equations of solid mechanics and appropriate coupling of the different parts involved in the physical set up of the problem. They then proposed a clever way to compute a numerical solution to their system of equations. This way, in [6], Ghergu and Ionescu were able to compute a city frequency constant: given the geometry and the specific physical constants of an idealized two dimensional city, they computed a frequency that leads to the coupling between vibrating buildings and underground seismic waves. This is quite an interesting first result, but it is limited in scope insofar as that city frequency constant was obtained by simply increasing the number of buildings at the expense of solving larger and larger systems. The main point of this study is to show that if instead we use a periodic Green's function and perform computations on a single period, we can compute coupling frequencies more efficiently. This allows for much faster computations, and in turn makes it possible to consider more complex geometries within a single period.

Here is an outline of this paper. In section 2 we introduce the physics of the problem under consideration. We restrict this study to anti plane shearing which allows us to use scalar displacement fields depending on two spatial variables. We take into account the mass densities and the shear rigidities of the buildings and of the underground. Tall buildings are then essentially modeled to be one dimensional with different values for displacements at their foundations and at their tops. These two different displacements are then related in a balance equation using the elastic moduli of the buildings. We then narrow our focus on time harmonic solutions, which we non dimensionalize. We obtain in this way a PDE which is just a Helmholtz equation in a half plane. At the boundary of the half plane a no force condition is imposed outside the building foundations, while at the foundations we obtain an integral differential equation expressing how the force acting on the buildings relates to the effective motion of these buildings.

In section 3 we cover the case where the number of buildings is finite and all the buildings are identical. This is a shorter section where we essentially recover results obtained previously by Ghergu and Ionescu, 6]. Their method relies on the symmetry of a boundary operator $T$ : we prove that $T$ is symmetric in Appendix A. In section 4 we cover the case of an infinite collection of identical buildings, arranged periodically. We examine how our new results relate to Ghergu and Ionecu's results as the number of buildings in their case grows large. Some technical details regarding our numerical method for computing resonant frequencies 
in the infinite, periodic, case are presented in appendix B.

Our new method using periodic domains and periodic Green's function results in much faster computations. This is the main reason why we are now able to study the case of buildings of variable height, mass, and, rigidity. We can assume that the number of buildings is infinite as long as a given pattern is repeated periodically. This is the subject of section 5 .

\section{The physics of the city-effect problem and non dimen- tionalization of the associated eigenvalue formulation}

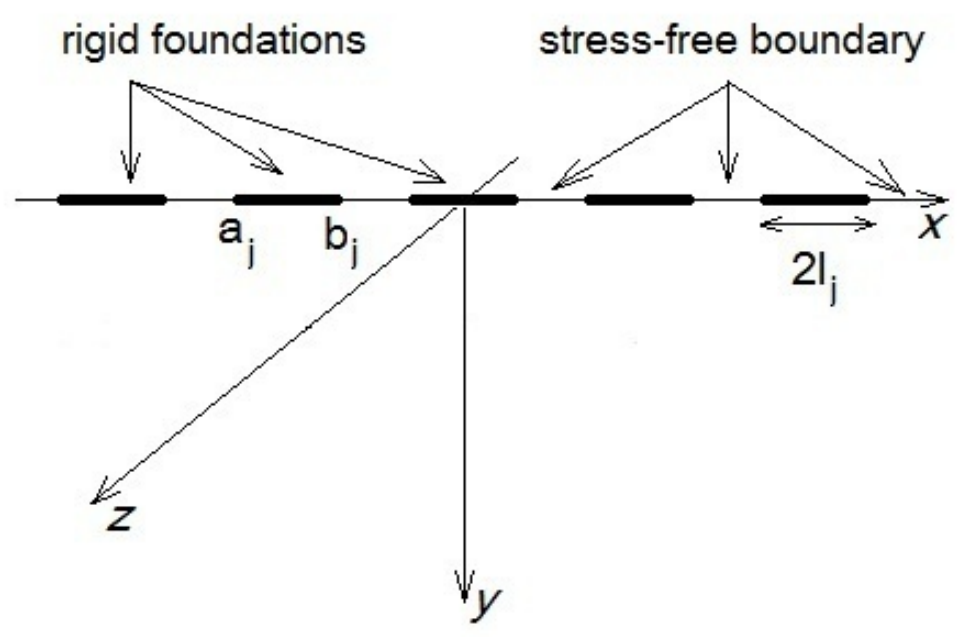

Figure 1: Model geometry: the building foundations lie on the intervals $\left[a_{j}, b_{j}\right]$ which appear in bold.

\subsection{Physical model}

We model the underground to be an elastic half-space $y>0$ in the $x y z$ space. We only consider the anti-plane shearing case: all displacements occur in the $z$ direction and are independent of $z$. We denote by $\Omega$ the half plane $y>0, z=0$. Consider $N$ buildings with width $2 l_{j}$ and height $h_{j}$ standing on the $x$ axis. In this study $N$ is either a finite number or it is infinite. In the first case the index $j$ will range from 1 to $N$, and in the second case $j$ will take all values in the set of integers $\mathbb{Z}$. The rigid building foundations are all located along the $x$-axis and denoted by $\Gamma_{j}=\left[a_{j}, b_{j}\right] \times\{0\}$ in $\Omega$. Let us introduce the following notations

- $\Gamma=\bigcup_{j=1}^{N} \Gamma_{j}$, if $N$ is finite, or $\Gamma=\bigcup_{j=-\infty}^{\infty} \Gamma_{j}$, if $N$ is infinite: the set of building foundations

- $\Gamma_{\text {free }}=\mathbb{R} \times\{0\} \backslash \Gamma$ : the stress free soil boundary 
- $w(t, x, y)$ : the scalar displacement field

The other physical parameters relevant to our problem are

- $\rho, \rho_{j}$ : the mass densities of the underground and of building $j$

- $S, S_{j}$ : the shear rigidities of the underground and of building $j$

- $\beta=\sqrt{S / \rho}, \beta_{j}=\sqrt{S_{j} / \rho_{j}}$ : the shear velocities of the underground and of building $j$

- $k_{j}$ : the elastic modulus of building $j$

- $u_{j}(t)$ : the displacement of the rigid building foundation $\Gamma_{j}$

- $v_{j}(t)$ : the displacement of the top of the building $j$

- $m_{1, j}, m_{0, j}$ : the mass at the top and at the foundation of building $j$

- $R_{j}(w)$ : the underground force acting on the building foundation $\Gamma_{j}$

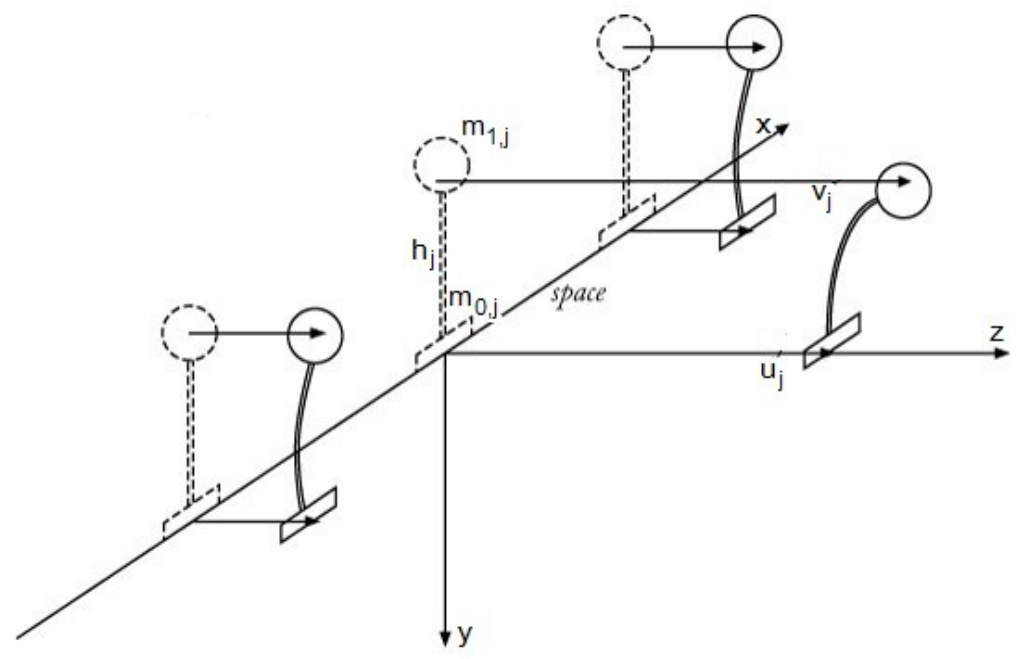

Figure 2: A sketch of city buildings subject to anti plane shearing. Here $N=3$. Building foundations are represented by rectangles and tops are represented by circles. Initial positions are sketched in dashed lines, displaced positions are sketched in solid lines. 
Using fundamental laws of solid physics, we obtain the following time dependent equations, where $t$ is the time variable,

$$
\begin{aligned}
\rho \ddot{w}(t) & =S \Delta w(t) \text { in } \Omega \times \mathbb{R}, \\
w(t, x, 0) & =u_{j}(t) \text { for all }(x, 0) \in \Gamma_{j}, \frac{\partial w}{\partial y}(t, x, 0)=0 \text { for all }(x, 0) \text { in } \Gamma_{\text {free }}, \\
m_{1, j} \ddot{v}_{j}(t) & =-k_{j}\left(v_{j}(t)-u_{j}(t)\right), \\
R_{j}(w) & =\int_{\Gamma_{j}} S \frac{\partial w}{\partial y}(t, s, 0) d s, \\
m_{0, j} \ddot{u}_{j}(t) & =R_{j}(w)+k_{j}\left(v_{j}(t)-u_{j}(t)\right) .
\end{aligned}
$$

Note that (1) is the wave equation for the displacement $w$; (2) shows that the displacement is constant for each rigid foundation and the space between the buildings is stress free; (3) comes from Newton's law of motion for the top of building $j$; equation (4) expresses continuity of forces between the underground and our one dimensional building foundations $\Gamma_{j}$; and (5) comes from Newton's law of motion for the bottom of building $j$.

\subsection{The associated spectral problem}

We focus on time harmonic solutions to the system (1.5). Accordingly, we set

$$
w(t, x, y)=\operatorname{Re}\left(\Phi(x, y) e^{-i \omega t}\right) .
$$

In other words $\omega>0$ is the associated frequency of a time harmonic vibration. Let us denote by $\alpha_{j}, \eta_{j}$ the displacements of the foundation and the top of each building. After substituting (6) in (1.5) we obtain

$$
\begin{aligned}
-S \Delta \Phi=\rho \omega^{2} \Phi \text { in } \Omega, \\
k_{j}\left(\eta_{j}-\alpha_{j}\right)=\omega^{2} m_{1, j} \eta_{j}, \quad-R_{j}(\Phi)-k_{j}\left(\eta_{j}-\alpha_{j}\right)=\omega^{2} m_{0, j} \alpha_{j}, \\
\Phi=\alpha_{j} \text { on } \Gamma_{j}, \frac{\partial \Phi}{\partial y}=0 \text { on } \Gamma_{\text {free }} .
\end{aligned}
$$

We now move on to non dimentionalize equations (7-9). We proceed exactly as in $[6$. We carefully cross checked all the corresponding calculations and our results agree with those from [6], save for the second identity in equation (8) of our present paper. This discrepancy is most likely due to a typographical error in 6]. We introduce a characteristic length $l$. The non-dimensional spatial coordinates are:

$$
x^{\prime}=\frac{x}{l}, y^{\prime}=\frac{y}{l}
$$

Accordingly, the non dimensional frequency comes out as

$$
\xi=\omega \frac{l}{\beta}
$$

From now on we will omit primes and write $x$ and $y$ in place of $x^{\prime}$ and $y^{\prime}$ for ease of notation. For each building $j$ we introduce the non dimensional parameters

$$
\gamma_{j}=\frac{m_{1, j}}{m_{0, j}}, f_{j}=\frac{l_{j}}{h_{j}}, c_{j}=\frac{l_{j}}{l}, r_{j}=\frac{\rho_{j}}{\rho}, \mathcal{B}_{j}=\frac{\beta_{j}}{\beta} .
$$


Note that $m_{1, j}, l_{j}, h_{j}, \rho_{j}$ are related by the equation

$$
m_{1, j}=2 l_{j} h_{j} \rho_{j},
$$

and $k_{j}$ is related to the shear rigidity $S_{j}$ through

$$
k_{j}=\frac{2 S_{j} l_{j}}{h_{j}} .
$$

After a long calculation we arrive at

$$
\eta_{j}=-\frac{\mathcal{B}_{j}^{2} f_{j}^{2} \alpha_{j}}{p_{j}\left(\xi^{2}\right)}, \text { where } p_{j}\left(\xi^{2}\right)=c_{j}^{2} \xi^{2}-\mathcal{B}_{j}^{2} f_{j}^{2}
$$

Further calculations lead to the system of equations

$$
\begin{gathered}
\Delta \Phi+\xi^{2} \Phi=0 \text { in } \Omega, \\
\frac{\partial \Phi}{\partial y}=0 \text { on } \Gamma_{\text {free }}, \\
q_{j}\left(\xi^{2}\right) \Phi(x, 0)=p_{j}\left(\xi^{2}\right) \int_{\Gamma_{j}} \frac{\partial \Phi}{\partial y}(s, 0) d s \text { for }(x, 0) \in \Gamma_{j},
\end{gathered}
$$

where

$$
q_{j}\left(\xi^{2}\right)=\frac{2 r_{j} c_{j}^{2} \xi^{2}}{f_{j}}\left(c_{j}^{2} \xi^{2}-\frac{\gamma_{j}+1}{\gamma_{j}} p_{j}\left(\xi^{2}\right)\right)
$$

Note that system (14) implies that $\Phi$ is constant on each $\Gamma_{j}$. We have denoted these constants by $\alpha_{j}$ : we will keep using this notation throughout this paper. Note also that equation (16) must hold for all integers $j$ between 1 and $N$, if there are $N$ buildings, or for all $j$ in $\mathbb{Z}$ if there are infinitely many buildings.

\section{The case of finitely many buildings which are all iden- tical and equally spaced}

In this case we assume that

- $N$ is finite

- all the physical parameters of the buildings $m_{1, j}, m_{0, j}, l_{j}, h_{j}, \rho_{j}, \beta_{j}$ are independent of $j$

- $b_{j}-a_{j}$ (the length of building $j$ ) and $a_{j+1}-b_{j}$ (the distance from building $j$ to building $j+1$ ) are independent of $j$

This was the only case considered in $[\underline{6}$. 


\subsection{Using linearity to reduce computational time}

Finding a solution to problem 14 16 becomes increasingly difficult as $N$ increases, however, Ghergu et al. were able to combine integral equation techniques to eigenvalues of relevant symmetric matrices in order to reduce computational time. Let us now overview their computational method. Fix $\alpha=\left(\alpha_{1}, . ., \alpha_{N}\right)$ in $\mathbb{R}^{N}$ and introduce the partial differential equation.

$$
\begin{aligned}
& \Delta \Psi+\xi^{2} \Psi=0 \text { in } \Omega, \\
& \Psi= \alpha_{j} \text { on } \Gamma_{j}, \frac{\partial \Psi}{\partial y}=0 \text { on } \Gamma_{\text {free }}, \\
& \frac{\partial \Psi}{\partial r}-i \xi \Psi=o\left(r^{-1 / 2}\right) \text { as } r=|x| \rightarrow+\infty .
\end{aligned}
$$

For any fixed $\xi>0$, problem $18-20$ is uniquely solvable: this can be shown using standard PDE techniques, see [11. We now introduce the $N \times N$ matrix $T\left(\xi^{2}\right)$ whose $k l$ entry is

$$
T\left(\xi^{2}\right)_{k l}=\operatorname{Re} \int_{\Gamma_{k}} \frac{\partial \Psi_{\xi, e_{l}}}{\partial y}(s, 0) d s
$$

where $e_{l}$ is the $l^{\text {th }}$ basis vector in $\mathbb{R}^{N}$, and $\Psi_{\xi, e_{l}}$ solves 18 for $\alpha=e_{l}$.

Gherghu et al. observed in [6] that the matrix $T\left(\xi^{2}\right)$ is symmetric. We provide a proof in Appendix A. Since $T\left(\xi^{2}\right)$ is symmetric, it can be diagonalized, and all its eigenvalues are real. For every $\xi^{2}>0$ we denote the eigenvalues of $T\left(\xi^{2}\right)$ by

$$
\tau_{1}\left(\xi^{2}\right) \leq \tau_{2}\left(\xi^{2}\right) \leq \ldots \leq \tau_{N}\left(\xi^{2}\right),
$$

and corresponding normalized eigenvectors $\theta_{1}\left(\xi^{2}\right), \ldots, \theta_{N}\left(\xi^{2}\right)$.

Since in the present case all the non-dimensional parameters $(12)$ are the same for all the buildings, the functions $p_{i}$ and $q_{i}$ do not depend on $i$ : they will therefore be simply denoted $p$ and $q$ in this section. Assume that $\xi$ is such that for some $i$

$$
p\left(\xi^{2}\right) \tau_{i}\left(\xi^{2}\right)=q\left(\xi^{2}\right) .
$$

Consider the corresponding solution $\Psi_{\xi, \theta_{i}\left(\xi^{2}\right)}$ to 1820 for $\alpha=\theta_{i}\left(\xi^{2}\right)$. Given that, $T\left(\xi^{2}\right) \theta_{i}\left(\xi^{2}\right)=$ $\tau_{i}\left(\xi^{2}\right) \theta_{i}\left(\xi^{2}\right)$, the coordinates of $\theta_{i}\left(\xi^{2}\right)$ are real and $\Psi$ solution to 18 20 is linear in $\alpha$, it follows that

$$
\operatorname{Re} \Psi_{\xi, \theta_{i}\left(\xi^{2}\right)}=\sum_{l=1}^{N}\left(\theta_{i}\left(\xi^{2}\right) \cdot e_{l}\right) \operatorname{Re} \Psi_{\xi, e_{l}}
$$

and

$$
\operatorname{Re} \int_{\Gamma_{k}} \frac{\partial \Psi_{\xi, \theta_{i}\left(\xi^{2}\right)}}{\partial y}(s, 0) d s=\tau_{i}\left(\xi^{2}\right) \theta_{i}\left(\xi^{2}\right) \cdot e_{k}
$$

for all $k$ from 1 to $N$. Now using relation 23 it follows that

$$
p\left(\xi^{2}\right) \operatorname{Re} \int_{\Gamma_{k}} \frac{\partial \Psi_{\xi, \theta_{i}\left(\xi^{2}\right)}}{\partial y}(s, 0) d s=q\left(\xi^{2}\right) \theta_{i}\left(\xi^{2}\right) \cdot e_{k},
$$

for all $k$ from 1 to $N$. We have thus found a wavenumber $\xi$ and boundary conditions $\alpha=\theta_{i}\left(\xi^{2}\right)$ such that 14 17 hold for $\Phi=\operatorname{Re} \Psi_{\xi, \theta_{i}\left(\xi^{2}\right)}$.

To find in practice a $\xi$ satisfying $(23)$ we fix $i$ and we proceed to solve numerically (23) as a non linear equation in $\xi$. The actual existence of a solution is a rather involved theoretical question. We address this question in a separate study, [11. 


\subsection{An integral equation for solving the system (18,20)}

Clearly, the crucial step in this symmetric matrix method is the ability to solve the Helmholtz problem 1820 . This is most easily done through an integral equation formulation. Accordingly, we set the solution to 18,20 to be

$$
\Psi(x, y)=\int_{\Gamma} G(x-s, y) \psi(s) d s,
$$

where $G$ is the usual fundamental solution of the Helmholtz equation: $G(x, y)=\frac{i}{4} H_{0}^{(1)}\left(\xi \sqrt{x^{2}+y^{2}}\right)$. We explain in [11] why formulation (24) is valid. Using potential theory we can argue that the function $\psi$ involved in 24 is in fact equal on $\Gamma$ to $\lim _{y \rightarrow 0^{+}}-2 \frac{\partial \Psi}{\partial y}(x, y)$. Due to equation 14 16. $\psi$ must satisfy

$$
\int_{\Gamma_{i}} G(x-s, y) \psi(s) d s=\alpha_{i}, \quad i=1, \ldots, N .
$$

We present in appendix a numerical method for solving integral equation (24).

\subsection{Numerical illustration for $N=1$}

We verify on an example that we can recover the numerical values obtained by Gherghu et al. in the case of a single building. In order to do this, we repeat a calculation from [6. More specifically, we treat the case relative to Figure 10 from [6] where the city and underground parameters were set to $l_{1}=1, b_{1}-a_{1}=0.4, l=l_{1}, \gamma_{1}=1.5, f_{1}=0.5, c_{1}=1$, $r_{1}=0.1, \mathcal{B}_{1}=1.5$. Note that in this case the matrix $T$ reduces to a scalar. Denote by $2 M$ the number of grid points for that building. For $M=10,20, \ldots, 100$, we solve the non linear equation in $\xi$ given by 222 where $i=N=1$ and $\tau_{1}\left(\xi^{2}\right)=\operatorname{Re} \int_{\Gamma_{1}} \frac{\partial \Psi_{\xi, e_{1}}}{\partial y}(s, 0)$. The numerical convergence of the solution as $M$ grows large is clearly observed in Figure 3 on this example we were able to replicate the results given by Figure 10 in 6 . We also note that for $M=5$ the numerical error appears to be under $3 \%$. 


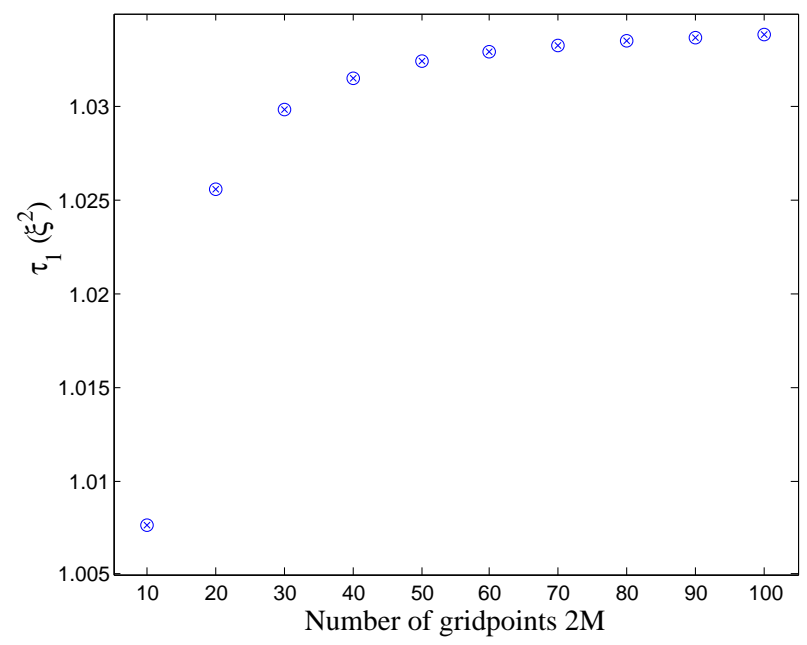

Figure 3: One-building city: $l_{b}=1, b_{1}-a_{1}=0.4, l=l_{1}, \gamma_{1}=1.5, f_{1}=0.5, c_{1}=1$, $r_{1}=0.1, \mathcal{B}_{1}=1.5$. Observed numerical convergence of the eigenvalue $\tau_{1}\left(\xi^{2}\right)$ as the number of gridpoints $2 M$ increases. Note that for $M=5$ the numerical error appears to be under $3 \%$.

\section{The large number of identical buildings case: conver- gence to periodic structures as $N$ grows large}

\subsection{Ghergu et al.'s large computations and our novel idea on how to drastically cut computational time}

In this case we assume that all the physical parameters of the buildings $m_{1, j}, m_{0, j}, l_{j}, h_{j}$, $\rho_{j}, \beta_{j}$ are independent of $j$ and $b_{j}-a_{j}$ and $a_{j+1}-b_{j}$ are independent of $j$, that is the length of foundations is constant, and the buildings are equally spaced. If $N$ is finite, that case was covered in [6]. Ghergu et al. were actually interested in identifying a collective behavior of a large set of buildings, which they called "city effect". To do so, they simply applied the computation outlined in the previous section and let the parameter $N$ grow large. Despite their brilliant idea consisting of using the matrix $T$ defined in the previous section, the computational time involved in their method can become prohibitive since it involves for each step of the search in $\xi$ solving a $2 M N$ by $2 M N$ linear system for $N$ different right hand sides. Our novel idea is to set directly $N=\infty$ and to introduce a periodic PDE.

Let us first introduce notations for our new periodic computational domain, where $2 P$ is the period in the $x$ direction

$$
\begin{aligned}
& \Gamma_{p e r}=\left[-l_{b}, l_{b}\right] \\
& \Gamma_{p e r}^{f r e e}=(-P, P) \backslash \overline{\Gamma_{p e r}} \\
& \Omega_{p e r}=(-P, P) \times(0, \infty)
\end{aligned}
$$

Accordingly, in place of 18 20 we now solve a periodic Helmholtz equation in a domain which is bounded in the $x$ direction. We formulate this partial differential equation as 
follows:

$$
\begin{gathered}
\Delta \Psi+\xi^{2} \Psi=0 \text { in } \Omega_{p e r}, \\
\Psi=1 \text { on } \Gamma_{p e r}, \frac{\partial \Psi}{\partial y}=0 \text { on } \Gamma_{p e r}^{f r e e} .
\end{gathered}
$$

augmented by the decay condition

$$
\frac{\partial \Psi}{\partial y}-i \xi \Psi=o\left(y^{-1 / 2}\right) \text { as } y \rightarrow+\infty,
$$

and the periodic boundary condition

$$
\Psi(-P, y)=\Psi(P, y) \text { for all } y>0
$$

The non linear equation in $\xi$ to be solved is now reduced to

$$
q\left(\xi^{2}\right)=p\left(\xi^{2}\right) \operatorname{Re} \int_{\Gamma_{p e r}} \frac{\partial \Psi}{\partial y}(s, 0) d s,
$$

Evidently this new computational technique relies on the ability to solve efficiently PDE 26 29). This can be done by setting

$$
\Psi(x, y)=\int_{\Gamma_{p e r}} G_{p e r}(x-s, y) \psi(s) d s,
$$

where $G_{p e r}$ is the adequate Green's function relative to the periodic problem $\sqrt[26]{29}$, and solving for $\psi$ the integral equation

$$
\int_{\Gamma_{p e r}} G_{p e r}(x-s, y) \psi(s) d s=1,
$$

where $x$ and $s$ are in $\Gamma_{p e r}$. Defining and computing $G_{p e r}$ is a vast subject: we discuss it in Appendix B.

\subsection{Numerical results and comparison to Ghergu et al.'s results}

It is instructive to compare values of resonant frequencies $\xi_{j}$ solving for some $j$ in $\{1, \ldots, N\}$, $p\left(\xi^{2}\right) \tau_{j}\left(\xi^{2}\right)=q\left(\xi^{2}\right)$ coupled to the $N$ identical, equally spaced, building problem 18 23) to values of resonant frequencies $\xi_{\text {per }}$ solving the periodic problem (26) We do that in the case where solutions are sought in the vicinity of the initial guess $\xi_{0}=1$. Our numerical simulations point to the following observations:

- $\xi_{j}$ is increasing in $j: \xi_{1} \leq . . \leq \xi_{j} \leq \xi_{j+1} \leq . . \leq \xi_{N}$

- all the resonant frequencies $\xi_{j}$ for $j$ in $\{1, . ., N\}$ vary within a narrow range. More precisely $\frac{\xi_{N}-\xi_{1}}{\xi_{N}}$ and $\frac{\xi_{N}-\xi_{1}}{\xi_{1}}$ are both small.

- $\xi_{1} \leq \xi_{\text {per }} \leq \xi_{N}$ (up to numerical accuracy)

- for smaller values of spacing between buildings $a_{j+1}-b_{j}, \xi_{\text {per }} \sim \xi_{N}$, and for larger values, $\xi_{\text {per }} \sim \xi_{1}$ 
Computed values of $\xi_{1}, \xi_{p e r}, \xi_{N}$ are given in Table $1 . N$ is here set to be 51 , and $M$ had to be set to as low as 5 , for computational time to be reasonable. The variable space is for the spacing between buildings $a_{j+1}-b_{j}$. The building half-widths are $l_{j}=1$. As previously, $l=l_{j}, \gamma_{j}=1.5, f_{j}=0.5, c_{j}=1, r_{j}=0.1, \mathcal{B}_{j}=1.5$.

\begin{tabular}{|c|c|c|c|c|c|c|c|}
\hline space & 0.5 & 1 & 1.3 & 1.4 & 1.5 & 2 & 3 \\
\hline$\xi_{1}$ & 0.7821 & 0.7990 & 0.8156 & 0.8264 & 0.8418 & $\mathbf{0 . 8 2 2 2}$ & $\mathbf{0 . 7 9 3 3}$ \\
\hline$\xi_{\text {per }}$ & $\mathbf{1 . 0 8 6 4}$ & $\mathbf{0 . 9 4 2 0}$ & 0.8873 & 0.8737 & 0.8619 & $\mathbf{0 . 8 2 2 5}$ & $\mathbf{0 . 7 9 3 4}$ \\
\hline$\xi_{N}$ & $\mathbf{1 . 0 8 4 4}$ & $\mathbf{0 . 9 4 0 8}$ & 1.0391 & 1.0514 & 1.0602 & 1.0635 & 0.9772 \\
\hline
\end{tabular}

Table 1: Numerical values for $\xi_{1}, \xi_{p e r}, \xi_{N}$ defined above. Here $N=51, M=5$ (2M is the number of grid points on each building). The variable space indicates the spacing between buildings. The building half-widths are $l_{j}=1$. As previously, $l=l_{j}, \gamma_{j}=1.5, f_{j}=0.5$, $c_{j}=1, r_{j}=0.1, \mathcal{B}_{j}=1.5$. 

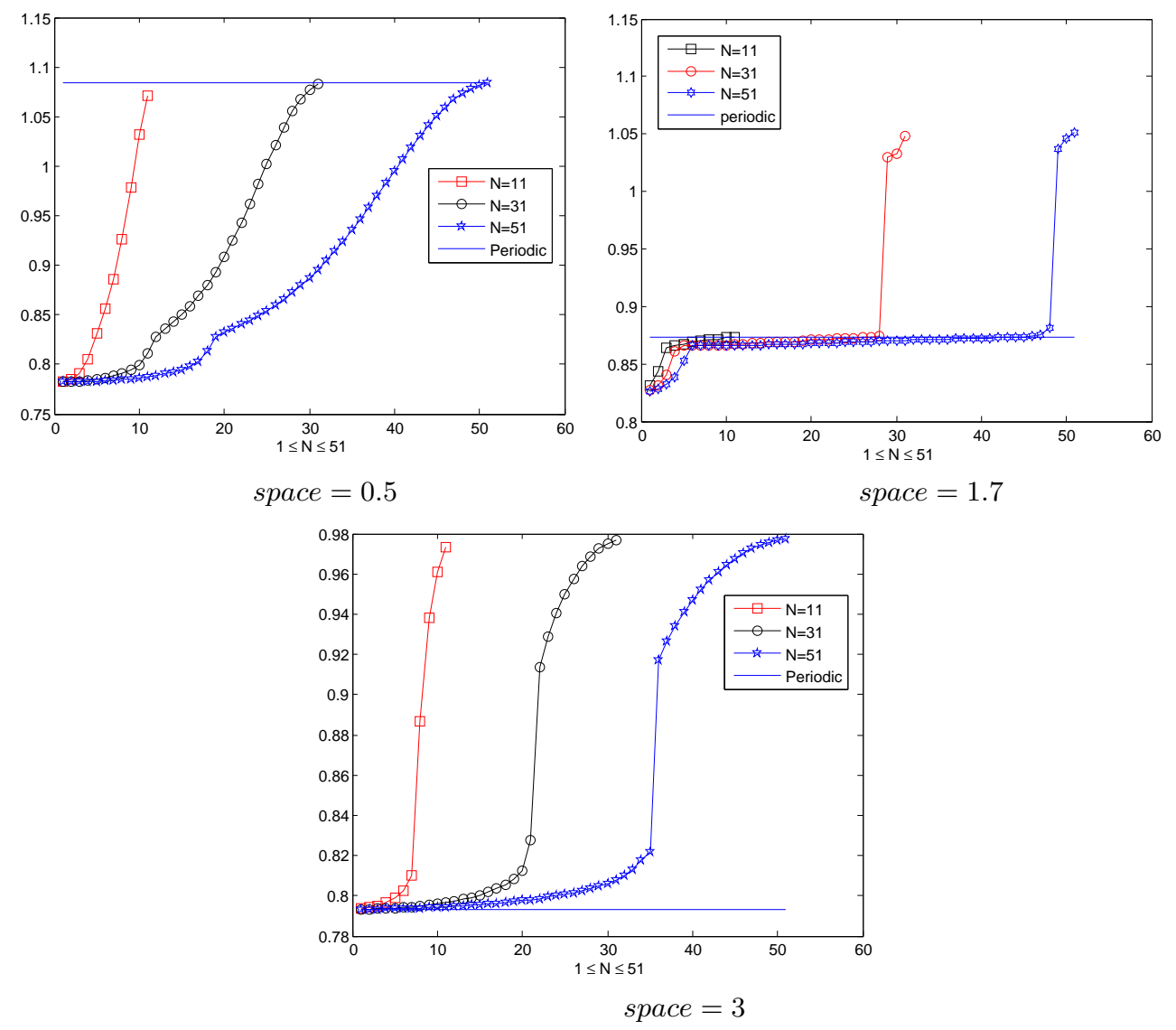

Table 2: Vertical axis: resonant frequencies $\xi_{j}$ solving $p\left(\xi^{2}\right) \tau_{j}\left(\xi^{2}\right)=q\left(\xi^{2}\right)$ coupled to the $N$ identical, equally spaced, building problem 1823 . Horizontal axis: value of integer $j$. Horizontal lines: value of $\xi_{p e r}$. Three cases corresponding to different spacings between buildings, $0.5,1.7$, and, 3 , are shown. For each spacing we examined the case where the total number of buildings $N$ is 11,31, or, 51. As previously, the physical parameters $l=l_{j}=1$, $\gamma_{j}=1.5, f_{j}=0.5, c_{j}=1, r_{j}=0.1, \mathcal{B}_{j}=1.5$.

\section{The case of sets of different buildings}

In this section the physical parameters $m_{1, j}, m_{0, j}, l_{j}, h_{j}, \rho_{j}, \beta_{j}$ may depend on the building $j$, and the lengths $b_{j}-a_{j}$ and $a_{j+1}-b_{j}$ too. Accordingly the two functions $p_{j}$ and $q_{j}$ defined in 17) may also depend on $j$, and consequently the symmetric matrix method used in section 4 is no longer applicable.

\subsection{Case where the number of buildings $N$ is finite}

In this case we have to solve for $\xi$ the system of $N$ non linear equations 


$$
\alpha_{i} q_{i}\left(\xi^{2}\right)=p_{i}\left(\xi^{2}\right) \operatorname{Re} \int_{\Gamma_{i}} \frac{\partial \Psi}{\partial y}(s, 0) d s, \quad i=1, \ldots, N
$$

where $\Psi$ and the real numbers $\alpha_{i}$ are coupled through the PDE 18 20. For each step in the search for $\xi$ satisfying (33), that PDE is solved by the same integral equation method as previously.

Let us now present some numerical results for this new case. We will vary building halfwidths $l_{j}$ and distances from one building to the next $a_{j+1}-b_{j}$. We choose the characteristic length $l=1$, so that that $c_{j}=l_{j}$. To facilitate comparison to previous cases, we will let $l_{j}$ vary while the other physical parameters will remain constant from one building to another and their values will be the same as previously. In other words, $\gamma_{1}=\gamma_{j}=1.5, f_{1}=f_{j}=0.5$, $r_{j}=r_{1}=0.1, \mathcal{B}_{j}=\mathcal{B}_{1}=1.5$. Clearly, since $l_{j}$ and $a_{j+1}-b_{j}$ are non constant, this is will lead to different functions $p_{j}$ and $q_{j}$ as $j$ varies.

Suppose that we can find a solution to $18,20,33$ such that for a particular index $j, \alpha_{j} \neq 0$. Then by linearity we can assume that $\alpha_{j}=1$. Let us now impose $\alpha_{j}=1$ in the search for a solution to $18,20,33)$. Our numerical simulations clearly indicate that solutions depend on the choice of such a $j$. In Table 3 we show solutions $(\xi, \alpha)$ for a 6 -building geometry sketched in Figure 4 , where $a=(0,1.3,3,4,5.4,6.8), b=(1,2.6,3.5,5,6.2,7.4)$ and $M=10$ ( $2 M$ was set to be the number of grid points per building in our numerical calculations). The values $\alpha_{j}$ of $\Psi(x, 0)$ on $\Gamma_{j}$ are shown as bar graphs, and coupling wavenumbers $\xi$ are given below each graph. We observe that the runs for $\alpha_{2}=1, \alpha_{3}=1$, and $\alpha_{6}=1$ all lead to the same eigenvalue $\xi=1.3660$. A closer look at Table 3 reveals that these three runs also lead, after rescaling, to the same eigenvector. In other words, the solutions in these three cases are clearly multiple of one another. The same remark can be made for the runs for $\alpha_{1}=1$ and $\alpha_{5}=1$.

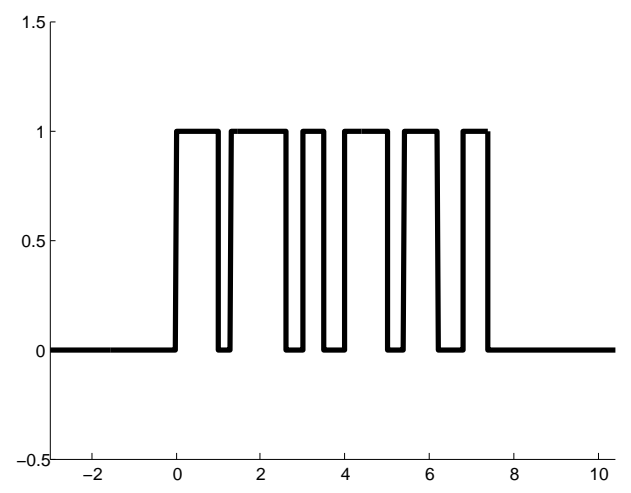

Figure 4: A sketch illustrating the relative size of the foundations and the spacing between the buildings for the 6 -building city defined by $a=(0,1.3,3,4,5.4,6.8), b=$ $(1,2.6,3.5,5,6.2,7.4)$. 


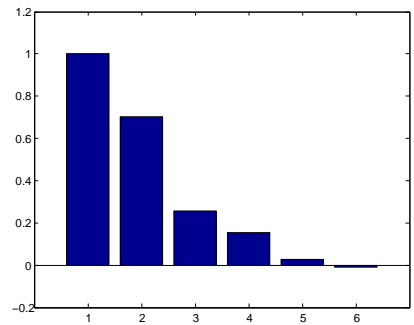

$\alpha_{1}=1, \xi=1.7301$

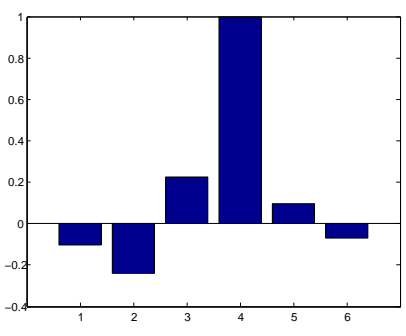

$\alpha_{4}=1, \xi=1.7784$

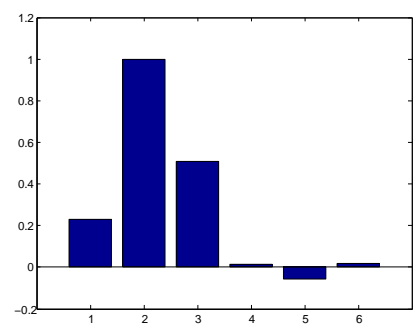

$\alpha_{2}=1, \xi=1.3660$

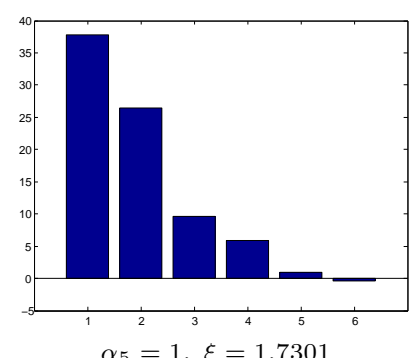

$\alpha_{5}=1, \xi=1.7301$

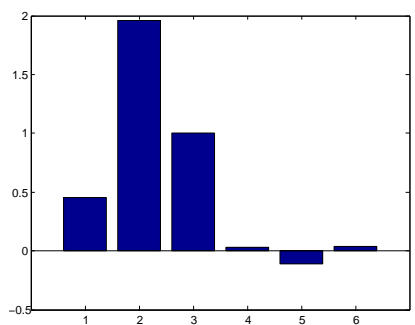

$\alpha_{3}=1, \xi=1.3660$

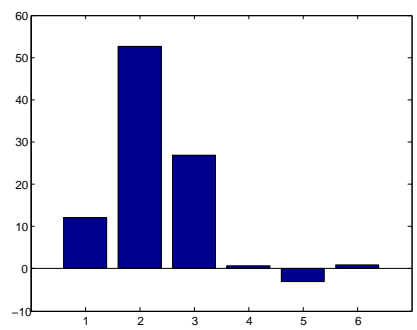

$\alpha_{6}=1, \xi=1.3660$

Table 3: Solutions for the 6-building city: $a=(0,1.3,3,4,5.4 ; 6.8), b=(1,2.6,3.5,5,6.2,7.4)$, $M=10$. The runs for $\alpha_{2}=1, \alpha_{3}=1$, and $\alpha_{6}=1$ all lead to the same eigenvalue $\xi=1.3660$. A closer look reveals that these three runs also lead, after rescaling, to the same eigenvector.

As expected, the solution for each case $\alpha_{j}=1$ is not unique. The results in Table 3 were obtained for an initial guess for the wavenumber $\xi_{0}=1$. Choosing instead $\xi_{0}=2.5$, we obtain the following:

if we impose $\alpha_{1}=1$, or $\alpha_{2}=1$, or $\alpha_{3}=1$, then our computation results in $\xi=1.7301$;

if we impose $\alpha_{4}=1$, or $\alpha_{5}=1$, then our computation results in $\xi=2.1861$;

if we impose $\alpha_{6}=1$, then our computation results in $\xi=2.8057$.

Let us now consider consider sets of buildings, referred to as cities, such that a pattern of buildings is repeated finitely many times. As previously $N$ denotes the total number of buildings and we now set $N=N_{c} B$ where $N_{c}$ is the number of repeated patterns or cells, and $B$ is the number of buildings in each cell. We now show numerical results for two examples of city geometry. They will be referred to as City 1 and City 2 .

1. City $1: \mathrm{B}=2,\left(a_{1}, a_{2}\right)=(-2.5,1.5),\left(b_{1}, b_{2}\right)=(-1.5,3), a_{j+2}-a_{j}=b_{j+2}-b_{j}=7.5$.

2. City 2: $\mathrm{B}=3,\left(a_{1}, a_{2}, a_{3}\right)=(0,2,5),\left(b_{1}, b_{2}, b_{3}\right)=(1.2,3,6.7), a_{j+3}-a_{j}=b_{j+3}-b_{j}=7$. 


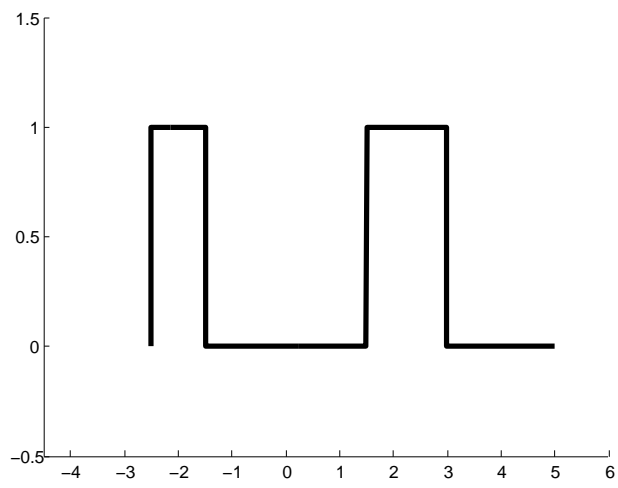

Figure 5: A sketch illustrating the relative size of the foundations and the spacing between the buildings for City 1 defined above.

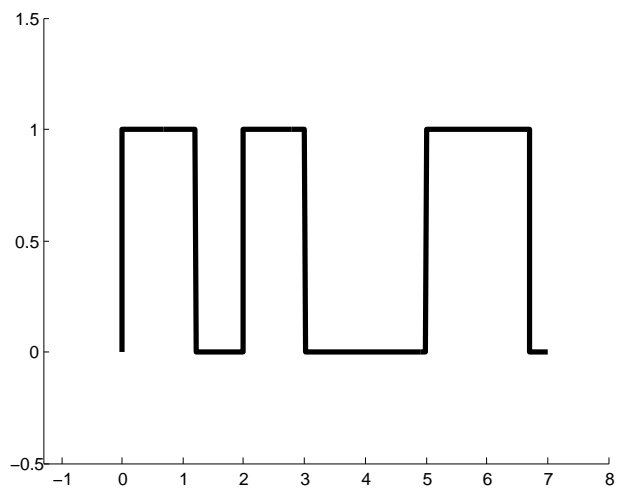

Figure 6: A sketch illustrating the relative size of the foundations and the spacing between the buildings for City 2 defined above.

Tables 4 and 5 show some computed solutions. They depend on the geometry of each city, including number of clusters $N_{c}$, on the the choice of building $j$ where the condition $\alpha_{j}=1$ is imposed and on the initial value $\xi_{0}$ for $\xi$ for the search of a solution to non linear equation (33). Here too, we observe that runs that differ in the choice of the index $j$ for which we impose $\alpha_{j}=1$ may eventually lead to the same eigenvector (after rescaling). In Table 4 this occurs for example, $\alpha_{6}=1, \alpha_{10}=1, \alpha_{12}=1$. 


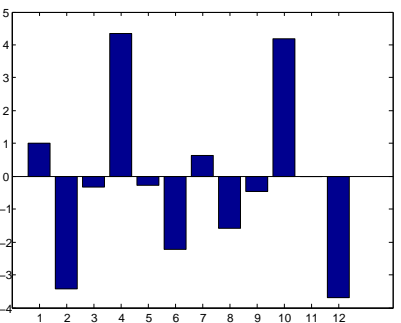

$\alpha_{1}=1, \xi=1.0778$

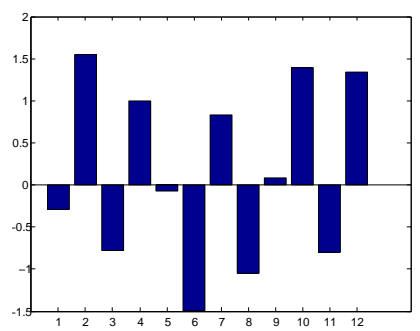

$\alpha_{4}=1, \xi=1.1489$

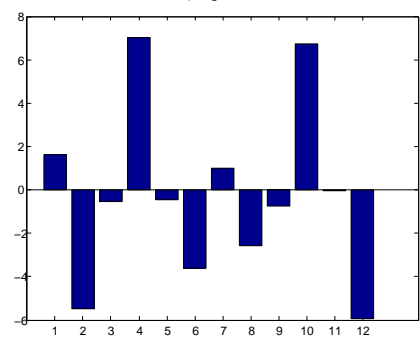

$\alpha_{7}=1, \xi=1.0778$

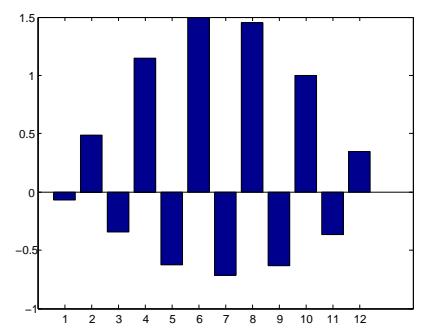

$\alpha_{10}=1, \xi=1.1584$

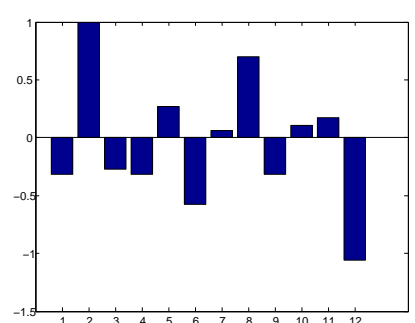

$\alpha_{2}=1, \xi=1.1212$

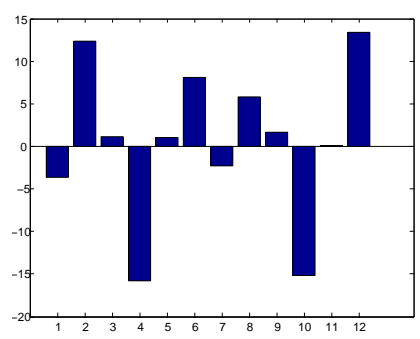

$\alpha_{5}=1, \xi=1.0778$

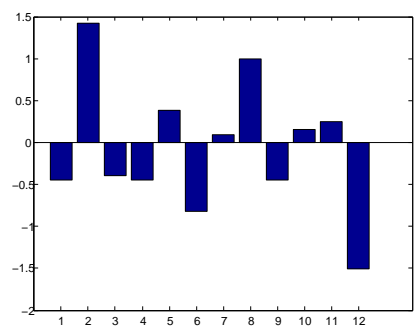

$\alpha_{8}=1, \xi=1.1212$

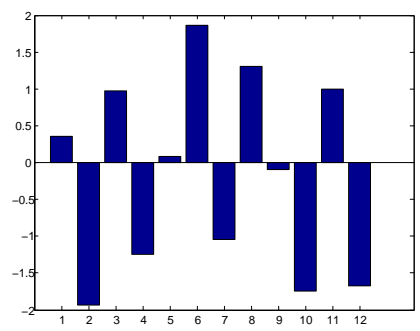

$\alpha_{11}=1, \xi=1.1489$

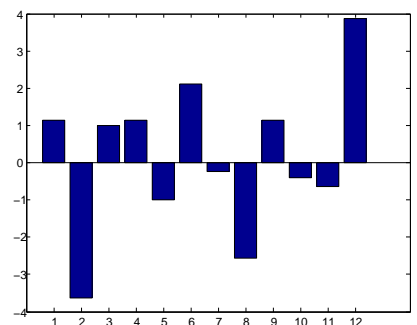

$\alpha_{3}=1, \xi=1.1212$

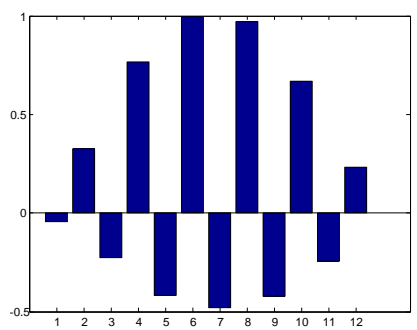

$\alpha_{6}=1, \xi=1.1584$

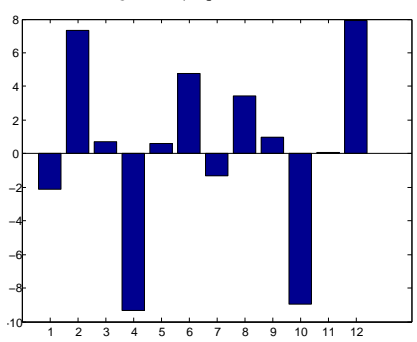

$\alpha_{9}=1, \xi=1.0778$

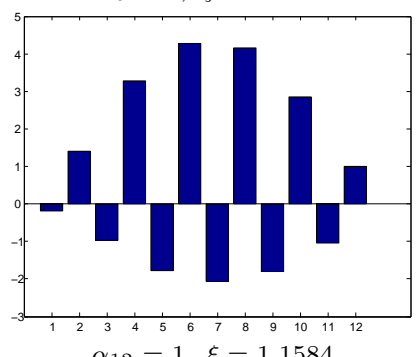

Table 4: City 1: number of cells is $N_{b}=6$. Number of buildings per cell is $B=2 . \quad M=5$ ( $2 M$ is the number of computational points per building). The foundation displacements $\alpha$ are depicted as bar graphs, $\xi$ are the coupling frequencies solving (33). In each case $\alpha_{j}=1$ is imposed, for the indicated $j$-th building. Note that runs that differ in the choice of the index $j$ for which we impose $\alpha_{j}=1$ may eventually lead to the same eigenvector (after rescaling). For example, $\alpha_{6}=1, \alpha_{10}=1, \alpha_{12}=1$. 


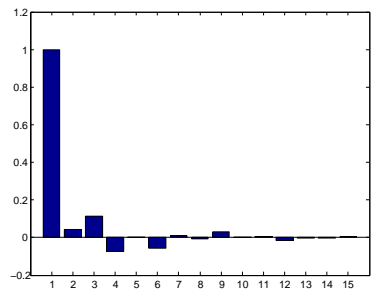

$\alpha_{1}=1, \xi=1.4119$

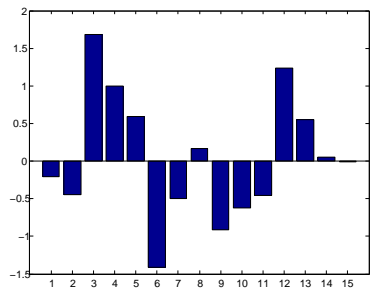

$\alpha_{4}=1, \xi=1.0043$

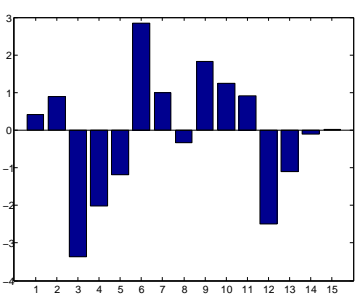

$\alpha_{7}=1, \xi=1.0043$

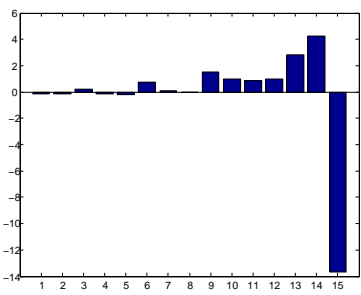

$\alpha_{10}=1, \xi=0.9837$

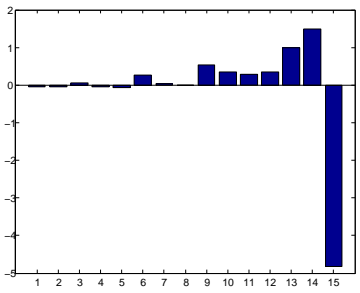

$\alpha_{13}=1, \xi=0.9837$

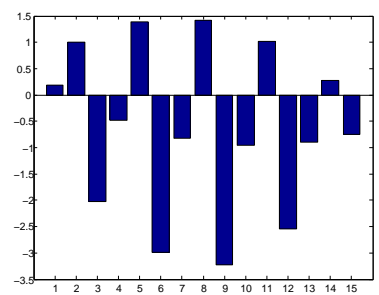

$\alpha_{2}=1, \xi=1.0349$

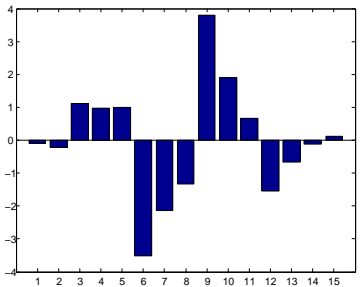

$\alpha_{5}=1, \xi=1.0048$

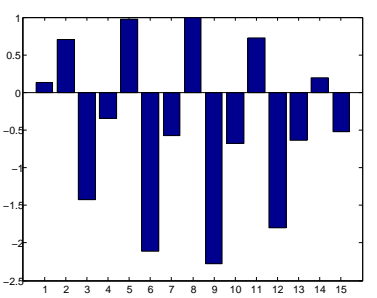

$\alpha_{8}=1, \xi=1.0349$

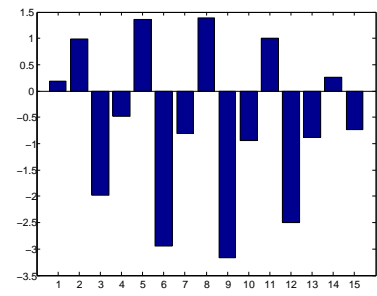

$\alpha_{11}=1, \xi=1.0349$

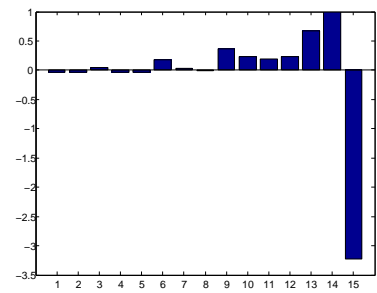

$\alpha_{14}=1, \xi=0.9837$

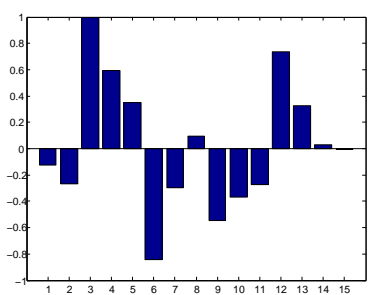

$\alpha_{3}=1, \xi=1.0043$

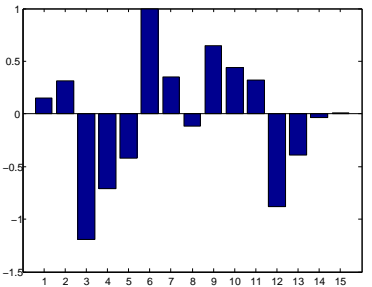

$\alpha_{6}=1, \xi=1.0043$

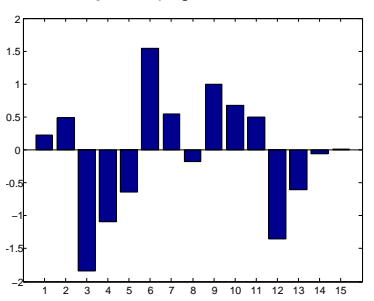

$\alpha_{9}=1, \xi=1.0043$

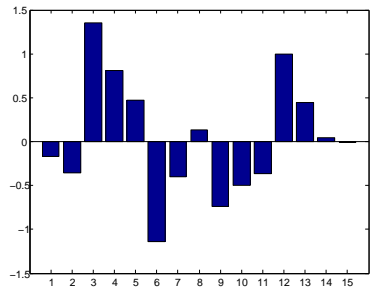

$\alpha_{12}=1, \xi=1.0043$

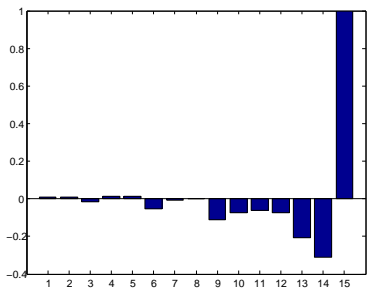

$\alpha_{15}=1, \xi=0.9837$

Table 5: City 2: number of cells is $N_{b}=5$. Number of buildings per cell is $B=3 . \quad M=5$ ( $2 M$ is the number of computational points per building). The foundation displacements $\alpha$ are depicted as bar graphs, $\xi$ are the coupling frequencies solving (33). In each case $\alpha_{j}=1$ is imposed, for the indicated $j$-th building. 


\subsection{Case of repeated patterns of buildings}

Assume that the number of buildings $N$ is now infinite and that there is a periodic pattern of buildings. We will denote by $B$ the number of buildings in the pattern (as previously), and by $2 P$ the length of each periodic cell. Accordingly,

- all the physical parameters of the buildings $m_{1, j}, m_{0, j}, l_{j}, h_{j}, \rho_{j}, \beta_{j}$ are periodic in $j$, with period $B$

- $a_{j+B}=a_{j}+2 P, b_{j+B}=b_{j}+2 P$

Assume that the foundations of the buildings $1, \ldots, B$ are included in some interval $\left[I_{1}, I_{2}\right]$, where $I_{2}-I_{1}=2 P$. Introduce the following notations: $\Omega_{p e r}=\left(I_{1}, I_{2}\right) \times(0, \infty), \Gamma_{p e r, j}=$ $\left[a_{j}, b_{j}\right], \Gamma_{p e r}^{f r e e}=\left(I_{1}, I_{2}\right) \backslash \cup_{j=1}^{B} \Gamma_{p e r, j}$. In this new case the analog of 2628 is

$$
\begin{gathered}
\Delta \Psi+\xi^{2} \Psi=0 \text { in } \Omega_{p e r}, \\
\Psi=\alpha_{j} \text { on } \Gamma_{p e r, j}, j=1, \ldots, N \frac{\partial \Psi}{\partial y}=0 \text { on } \Gamma_{p e r}^{\text {free }} .
\end{gathered}
$$

augmented by the decay condition (28). The corresponding periodic boundary condition is

$$
\Psi\left(I_{1}, y\right)=\Psi\left(I_{2}, y\right) \text { for all } y \geq 0
$$

To find coupling frequencies for the underground and buildings system we have to solve the system of $B$ non linear equations

$$
\alpha_{j} q_{j}\left(\xi^{2}\right)=p_{j}\left(\xi^{2}\right) \operatorname{Re} \int_{\Gamma_{p e r, j}} \frac{\partial \Psi}{\partial y}(s, 0) d s, \quad j=1, \ldots, B
$$

where these equations are coupled through the PDE $(28,34,36)$. As previously, one of the $\alpha_{j}$ 's in 35 may be set to 1 , while the others will have to be determined.

Let us now examine a numerical example. The patterns of buildings are similar to those from the previous section, to facilitate comparison. We discuss two cases, City 1-per and City 2-per (where "per" is for periodic):

- City 1-per: $B=2, P=7.5,\left(a_{1}, a_{2}\right)=(-2.5,1.5), \quad\left(b_{1}, b_{2}\right)=(-1.5,3)$,

- City 2-per: $B=3, P=7,\left(a_{1}, a_{2}, a_{3}\right)=(0,2,5), \quad\left(b_{1}, b_{2}\right)=(1.2,3,6.7)$.

Accordingly the geometry of each periodic cell of these two cities is the same as the ones sketched in Figures 5 and 6. As previously, we pick $\gamma_{1}=\gamma_{j}=1.5, f_{1}=f_{j}=0.5, r_{j}=$ $r_{1}=0.1, \mathcal{B}_{j}=\mathcal{B}_{1}=1.5$, but the lengths of the building foundations, $b_{j}-a_{j}$ are variable. The table below gives computed values of coupling frequencies $\xi$, given an initial search value $\xi_{0}$. We varied $2 M$, the number of grid points on each building to illustrate numerical convergence.

1. City 1-per

$$
\begin{aligned}
& \xi_{0}=1, M=5: \xi=1.1594, \alpha=(1,-2.1171), \\
& \xi_{0}=1, M=10: \xi=1.1583, \alpha=(1,-2.1222), \\
& \xi_{0}=1, M=20: \xi=1.1580, \alpha=(1,-2.1241) .
\end{aligned}
$$


2. City 2-per

$$
\begin{aligned}
& \xi_{0}=1, M=5: \quad \xi=1.0420, \alpha=(1,-1.5703,3.5458), \\
& \xi_{0}=1, M=10: \quad \xi=1.0382, \alpha=(1,-1.5103,3.4610), \\
& \xi_{0}=1, M=20: \xi=1.0368, \alpha=(1,-1.4874,3.4288) .
\end{aligned}
$$

Next we report that given a pattern of $B$ buildings, repeated $N_{c}$ times, some computed coupling frequencies exhibit a convergence trend as $N_{c}$ grows large, and the limit value equals a coupling frequency for the periodic problem. Let us examine the case of the geometry given by City 1. In the periodic case City 1-per we found for $M=5$ and the initial values $\xi_{0}=1, \alpha_{1}=1$, the final values $\xi_{\text {per }}=1.1594, \alpha_{2}=-2.1222$. This is clearly close to the two cases shown in Table 4 for, say, $\alpha_{6}=1$. If we keep increasing the number of clusters $N_{c}$ we find at least one solution $\xi$ which approaches $\xi_{\text {per }}$, as shown in Table 6. We also notice convergence, in some sense, of the parameter $\alpha$. In Table 8 we sketched the computed value $\left(\alpha_{1}, \alpha_{2}\right)=(1,-2.1171)$ for City 1-per (when the initial guess for $\xi$ is $\left.\xi_{0}=1\right)$, which is clearly close to a multiple of $\left(\alpha_{7}, \alpha_{8}\right)$ obtained in the case $\alpha_{6}=1$ in Table 4.

Similar observations can be made in the case of the geometry of City 2: see Table 7 . This time $\left(\alpha_{1}, \alpha_{2}, \alpha_{3}\right)$ computed in the periodic case, given in Table 8 compares to $\left(\alpha_{7}, \alpha_{8}, \alpha_{9}\right)$ in Table 5 , case $\alpha_{8}=1$.

\begin{tabular}{|c|c|c|c|c|}
\hline & $N_{c}=4$ & $N_{c}=5$ & $N_{c}=6$ & Periodic \\
\hline$\xi$ & 1.1572 & 1.1579 & 1.1584 & 1.1594 \\
\hline
\end{tabular}

Table 6: Convergence to $\xi_{p e r}$, for the geometry given by City 1 . $M=5$.

\begin{tabular}{|c|c|c|c|c|c|}
\hline & $N_{c}=2$ & $N_{c}=3$ & $N_{c}=4$ & $N_{c}=5$ & Periodic \\
\hline$\xi$ & 1.0116 & 1.02160 & 1.0299 & 1.0349 & 1.0420 \\
\hline
\end{tabular}

Table 7: Convergence to $\xi_{p e r}$, for the geometry given by City 2. $M=5$.
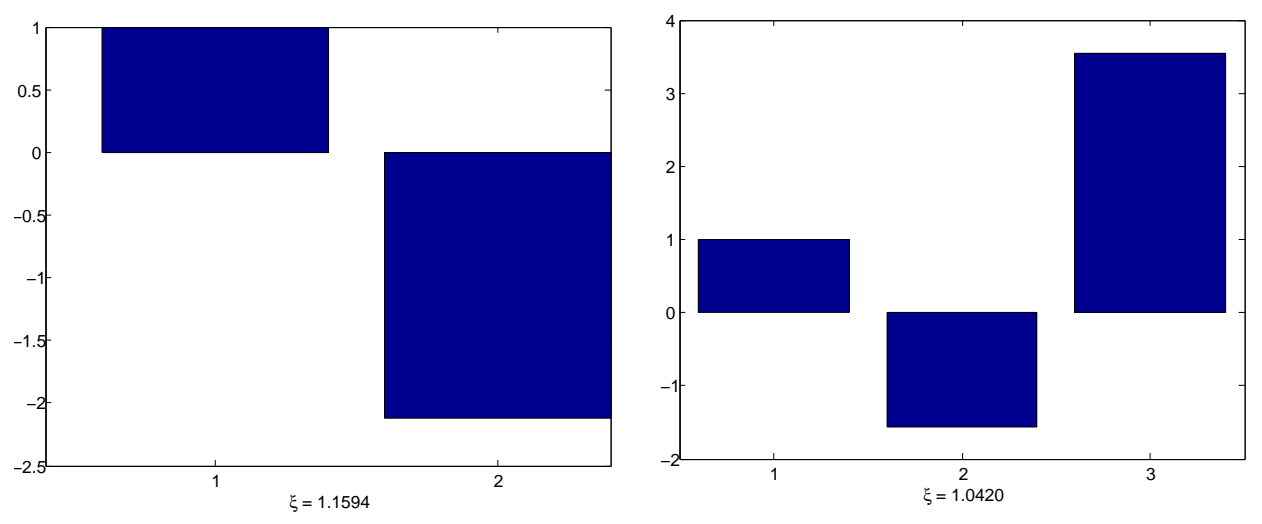

Table 8: Left: computed values of $\left(\alpha_{1}, \alpha_{2}\right)$ for the periodic case City 1-per. Right: computed values of $\left(\alpha_{1}, \alpha_{2}, \alpha_{3}\right)$ for the periodic case City 2-per. 


\subsection{Conclusion and perspectives}

Using a model involving vibrating tall buildings and harmonic elastic (anti plane) displacements of the underground, we have introduced in this paper methods for computing frequencies that achieve the coupling of these two vibrations. Earlier computations by Ghergu and Ionescu, [ 6], were limited to the case of a finite set of identical, equally spaced buildings. Their results are quite instructive since they point to a collective response of buildings to seismic waves, a phenomenon that authors have named "city-effect", 4]. We noted, however, that the method for finding coupling frequencies introduced by Ghergu and Ionescu becomes quickly computationally expensive as the number of buildings grows large, and may be hard to extend to a fully three dimensional setting. Given that weakness, we have therefore resorted to the use of periodic formulations. We have first obtained results that coincide with those of Ghergu et al.'s, but that can be obtained at a much lower computational cost. From there we have shown how our method can be extended to repeated sets of non identical buildings: more realistic city lay outs can thus be modeled. Interestingly, in the case of non identical buildings, our simulations indicate that the response to this coupling phenomenon may differ drastically from one building to another.

Our next endeavor, which will be the subject of a forthcoming publication, will be to study fully three dimensional models. Generalizing our work to that case will certainly prove to be quite challenging since the 3D elastic half space Green's tensor is already very involved, and we would need to compute its periodic analog.

\section{Appendix A: proof of symmetry for matrix $T$ defined by formula (21)}

Let $\psi_{k}$ solve 1820 with $\alpha_{j}=1$, if $j=k$, and $\alpha_{j}=0$ otherwise. It suffices to show that

$$
\int_{\Gamma_{i}} \Psi_{i} \frac{\partial \Psi_{j}}{\partial y} d s=\int_{\Gamma_{j}} \Psi_{j} \frac{\partial \Psi_{i}}{\partial y} d s
$$

Denote by $D$ the set $\left\{(x, y): x^{2}+y^{2} \leq r^{2}, y \geq 0\right\}$. Due to Green's theorem,

$$
\int_{D}\left(\Psi_{i} \Delta \Psi_{j}-\Psi_{j} \Delta \Psi_{i}\right) d x d y=\int_{\partial D}\left(\Psi_{i} \frac{\partial \Psi_{j}}{\partial \nu}-\Psi_{j} \frac{\partial \Psi_{i}}{\partial \nu}\right) d s
$$

where $\nu$ is the unit normal vector to $\partial D$ pointing outward. Using 1820 it follows that

$$
\begin{aligned}
\int_{\Gamma_{i}} \Psi_{i} \frac{\partial \Psi_{j}}{\partial y} d s-\int_{\Gamma_{j}} \Psi_{j} \frac{\partial \Psi_{i}}{\partial y} d s & =\int_{\{|x|=r, y>0\}} \Psi_{i} \frac{\partial \Psi_{j}}{\partial r}-\Psi_{j} \frac{\partial \Psi_{i}}{\partial r} d s \\
& =\int_{\{|x|=r, y>0\}}\left(\Psi_{j}-\Psi_{i}\right) o\left(r^{-1 / 2}\right) d s
\end{aligned}
$$

Due the decay of the fundamental solution of the Helmholtz equation in a half plane, we have that $\Psi_{j}=O\left(r^{-1 / 2}\right)$ and $\Psi_{i}=O\left(r^{-1 / 2}\right)$, so if we let $r \rightarrow \infty$ we arrive at identity (38). 


\section{Appendix B: the numerical solutions to equations (25) and $(32)$}

\subsection{The free space case: equation (25)}

It is well known in the literature that $\psi$ solution to 25 must have square root singularities at the edges of $\Gamma_{j}$, therefore we set

$$
\psi(s)=\frac{\phi(s)}{\sqrt{\left(s-a_{j}\right)\left(b_{j}-s\right)}}, \text { where } s \in\left(a_{j}, b_{j}\right), 1 \leq j \leq N,
$$

and $\phi$ is a smooth function in $\left[a_{j}, b_{j}\right]$. Recalling (24) we have

$$
\Psi(x, y)=\frac{i}{4} \sum_{j=1}^{N} \int_{a_{j}}^{b_{j}} H_{0}^{(1)}\left(\xi \sqrt{(x-s)^{2}+y^{2}}\right) \psi(s) d s,
$$

We use potential theory to assert that $\lim _{t \rightarrow 0^{+}} \frac{\partial \Psi}{\partial y}(s, t)=-\frac{1}{2} \psi(s)$ for all $a_{j}<s<b_{j}$. To employ the same numerical mesh for each building foundation $\Gamma_{j}$, we set for $t$ in $[-1,1]$

$$
s=g_{j}(t)=\frac{b_{j}-a_{j}}{2} t+\frac{b_{j}+a_{j}}{2}
$$

Substituting in 400 we obtain

$$
\frac{i}{4} \sum_{j=1}^{N} \int_{-1}^{1} H_{0}^{(1)}\left(\xi\left|x-g_{j}(t)\right|\right) \frac{\varphi\left(g_{j}(t)\right)}{\sqrt{1-t^{2}}} d t=\alpha_{k}, 1 \leq k \leq N, x \in \Gamma_{k} .
$$

We then solve for $\varphi\left(g_{j}(t)\right)$ following the numerical method introduced in the appendix of [6. Here we just recall that this numerical method relies on the fact that (see [1])

$$
\frac{i}{4} H_{0}^{(1)}(z)=A(z) \ln \frac{z}{2}+B(z),
$$

for any non zero complex number $z$, where $A$ and $B$ are two entire functions.

\subsection{The periodic case: equation $(32)$}

The periodic Green's function relative to problem $[26+29$ ) can be written out as

$$
G_{p e r}(x, y)=\sum_{n=-\infty}^{\infty} G(x-2 n P, y)
$$

The analog of decomposition 43 is now

$$
G_{p e r}(x, y)=A\left(\xi \sqrt{x^{2}+y^{2}}\right) \ln \frac{\xi \sqrt{x^{2}+y^{2}}}{2}+\tilde{B}(x, y),
$$


where

$$
\tilde{B}(x, y)= \begin{cases}\sum_{n=-\infty}^{\infty} G(x-2 n P, y)-A\left(\xi \sqrt{x^{2}+y^{2}}\right) \ln \frac{\xi \sqrt{x^{2}+y^{2}}}{2}, & \text { if } x^{2}+y^{2} \neq 0, \\ \frac{i \pi-2 \mathcal{C}}{4 \pi}+\sum_{n \neq 0} G(-2 n P, 0), & \text { otherwise }\end{cases}
$$

and $\mathcal{C}$ is the Euler constant. Note that $\tilde{B}$ is real analytic in $(x, y)$ : this is due to the asymptotics of the Hankel function $H_{0}^{(1)}$, see [1]. At this stage we see that the numerical method for the integral equation for the free space case 25 ) can be extended to the integral equation 232 for the periodic case: all we need to do is to replace $B\left(\xi\left|x-g_{j}(t)\right|\right)$ by $\tilde{B}\left(\xi\left(x-g_{j}(t)\right), 0\right)$. It is crucial to be able to compute $\tilde{B}(0,0)$ for this numerical method to be applicable: see [10] for a more detailed account of a comparable calculation and computational method for a related Green's function.

As explained above, we need to be able to efficiently compute the two slowly convergent sums $\sum_{n=-\infty}^{\infty} G(x-2 n P, y)$ and $\sum_{n \neq 0} G(-2 n P, 0)$. The first sum can be efficiently computed by Ewald's method. For that subject, we refer the reader to 9 . We did not find in the literature any results on the computation of the second sum, however, it can be inferred from the first sum. Here is how: setting in this appendix only, $p=\pi / P, r_{m}=\sqrt{(x-2 m P)^{2}+y^{2}}$ and

$$
\begin{gathered}
\gamma_{m}=\sqrt{m^{2} p^{2}-\xi^{2}}, \text { if } m^{2} p^{2}-\xi^{2}>0, \\
\gamma_{m}=i \sqrt{-m^{2} p^{2}+\xi^{2}}, \text { if } m^{2} p^{2}-\xi^{2}<0 .
\end{gathered}
$$

Then applying Ewald's formula (see [9]),

$$
\begin{gathered}
G_{p e r}(x, y)=\frac{1}{8 P} \sum_{m=-\infty}^{\infty} \frac{e^{i p m x}}{\gamma_{m}}\left[e^{\gamma_{m} y} \operatorname{erfc}\left(\frac{\gamma_{m} P}{a}+\frac{a y}{2 P}\right)+e^{-\gamma_{m} y} \operatorname{erfc}\left(\frac{\gamma_{m} P}{a}-\frac{a y}{2 P}\right)\right] \\
+\frac{1}{4 \pi} \sum_{m=-\infty}^{\infty} \sum_{n=0}^{\infty} \frac{1}{n !}\left(\frac{\xi P}{a}\right)^{2 n} E_{n+1}\left(\frac{a^{2} r_{m}^{2}}{4 P^{2}}\right),
\end{gathered}
$$

where $a>0$ is called "splitting parameter",

$$
\operatorname{erfc}(z)=\frac{2}{\sqrt{\pi}} \int_{z}^{\infty} e^{-t^{2}} d t
$$

is the complementary error function, and

$$
E_{n}(z)=\int_{1}^{\infty} t^{-n} e^{-z t} d t
$$

is the exponential integral. Note that the present method is valid only if $\gamma_{m} \neq 0$ for all integers $m$. (Note that if $\gamma_{m}=0$ for some $m$ then the system of equations $(26) 29$ is non 
uniquely solvable).

We now proceed to find a new series formula for the expression $\sum_{m \neq 0} \frac{i}{4} H_{0}^{(1)}\left(\xi r_{m}\right)$ for $(x, y)=$ $(0,0)$. The only singular term in 47 as $x=y=0$ is $E_{1}\left(\frac{a^{2} r_{0}^{2}}{4 P^{2}}\right)$ : it appears in the second sum for $m=n=0$. Note that

$$
E_{1}\left(\frac{a^{2} r_{0}^{2}}{4 P^{2}}\right)=-\left\{\mathcal{C}+\ln \frac{a^{2} r_{0}^{2}}{4 P^{2}}+\sum_{k=1}^{\infty} \frac{(-1)^{k}}{k \cdot k !}\left(\frac{a^{2} r_{0}^{2}}{4 P^{2}}\right)^{k}\right\},
$$

according to formula 5.1.11 in [1]. We may also expand $\frac{i}{4} H_{0}^{(1)}\left(\xi r_{0}\right)$ as follows

$$
\begin{aligned}
\frac{i}{4} H_{0}^{(1)}\left(\xi r_{0}\right) & =\frac{i}{4}\left(J_{0}\left(\xi r_{0}\right)+i Y\left(\xi r_{0}\right)\right) \\
& =\frac{i}{4} J_{0}\left(\xi r_{0}\right)-\frac{1}{2 \pi}\left(\ln \frac{\xi r_{0}}{2}+\mathcal{C}\right) J_{0}\left(\xi r_{0}\right)+\frac{1}{2 \pi} \sum_{m=1}^{\infty} a_{m} \frac{(-1)^{m}}{(m !)^{2}}\left(\frac{\xi r_{0}}{2}\right)^{2 m}
\end{aligned}
$$

where $a_{m}=\sum_{j=1}^{m} \frac{1}{j}$ (see formulas 9.1.13 in [1]).

Finally, we substitute $E_{1}$ in $(47)$ by (50), and we subtract (51). We note that the logarithmic singularities in $\ln r_{0}$ cancel out. As $x=y=0, r_{m}=2|m| \vec{P}$ and we obtain,

$$
\begin{aligned}
\sum_{n \neq 0} G(-2 n P, 0)=\frac{1}{4 P} \sum_{m=-\infty}^{\infty} \frac{1}{\gamma_{m}} \operatorname{erfc}\left(\frac{\gamma_{m} P}{a}\right)+ & \frac{1}{4 \pi} \sum_{m \neq 0} \sum_{n=0}^{\infty} \frac{1}{n !}\left(\frac{\xi P}{a}\right)^{2 n} E_{n+1}\left(a^{2} m^{2}\right) \\
& +\frac{1}{4 \pi} \sum_{n=1}^{\infty} \frac{1}{n n !}\left(\frac{\xi P}{a}\right)^{2 n}+\frac{1}{2 \pi} \ln \frac{\xi P}{a}
\end{aligned}
$$

\section{References}

[1] M. Abramovitz, I. A. Stegun, Handbook of Mathematical Functions. 10 $0^{\text {th }}$ Printing, National Bureau of Standards, 1972.

[2] P. Y. Bard, J. L. Chazelas, Ph. Gueguen, M. Kham, J. F. Semblat, Site-City Interaction, in: C. S. Oliveira, A. Roca, X. Coula (eds.), Assessing and Managing Risk, 91-114. Springer, 2006.

[3] S. C. Bradford, J. F. Clinton, J. Favela, T. H. Heaton, Results of Millikan library forced vibration testing, Report No. EERL 2004-3, Feb. 2004.

[4] C. Boutin, P. Roussillon, Assessment of the Urbanization Effect on Seismic Response. Bull. Seism. Soc. Am., vol. 94, No. 1 (2004) 251-268.

[5] S. Erlingsson, A. Bodare, Live load induced vibrations in Ullevi Stadium - dynamic underground analysis, underground Dyn. and Earthquake Eng., vol. 15, Issue 3 (1996) 171-188. 
[6] M. Ghergu, I. R. Ionescu, Structure-underground-structure coupling in seismic excitation and "city-effect". Int. J. Eng. Sci. 47 (2009) 342-354.

[7] http://earthquake.usgs.gov/earthquakes/world/events/1985_09_19.php

[8] H. Kanamori, J. Mori, B. Sturtevant, D. L. Anderson, T. Heaton, Seismic excitation by space shuttles, Shock waves, 2 (1992) 89-96.

[9] C. M. Linton, The Green's function for the two-dimensional Helmholtz equation in periodic domains. J. Engng. Math. 33 (1998) 377-402.

[10] D. Volkov, D. T. Papageorgiou, P. G. Petropoulos, Accurate and Efficient Boundary Integral Methods for Electrified Liquid Bridge Problems, SIAM J. Sci. Comput., 26 (6) (2005) 2102-2132.

[11] D. Volkov and S. Zheltukhin, Existence of Frequency Modes Coupling Seismic Waves and Vibrating Tall Buildings, submitted. (submission available on arXiv.org)

[12] A. Wirgin, P.-Y. Bard, Effects of buildings on the duration and amplitude of ground motion in Mexico City, Bull. Seism. Soc. Am., vol. 86 (1996) 914-920. 